\author{
Igor Vladimirovich Vasilev ${ }^{1}{ }^{\circ}$, Igor Sergeevich Mamenko ${ }^{1}{ }^{-}$, Anna Valerievna Makarova ${ }^{1}$, \\ Olga Pavlovna Sokolova', Vladimir Fedorovich Lee', Mahmud Mustafa Mortada' ${ }^{1}{ }^{1}$, \\ Tatiana Alexandrovna Novickaya ${ }^{1,2}$, Piotr Kazimirovich Yablonskii ${ }^{1,2}$ \\ 'Saint-Petersburg State Research Institute of Phthisiopulmonology, Saint-Petersburg, Russian Federation \\ ${ }^{2}$ Saint-Petersburg State University, Medical Faculty, Saint-Petersburg, Russian Federation
}

\title{
Probe-based confocal laser endomicroscopy in COVID-19
}

\begin{abstract}
Probe-based confocal laser endomicroscopy ( $\mathrm{pCLE}$ ) is a method that produces microscopic imaging of a lung tissue during bronchoscopy. We report a case ot a patient with negative nasopharyngeal swabs and suspected lung cancer who underwent pCLE. The diagnosis of COVID-19 was confirmed by PCR analyses of lavage fluid and transbrohial biopsy. The pCLE image shows density of alveolar thickened fibres, disorganization of elastin network, and multiple large drops of intraalveolar secretions. As far as we know, this is the first pCLE image discribed in patient with COVID-19 at that moment.
\end{abstract}

Key words: confocal laser endomicroscopy, optical biopsy, COVID-19, SARS-CoV-2

Adv Respir Med. 2021; 89: 456-459

\section{Introduction}

Coronavirus disease 2019 (COVID-19) is a type of lower respiratory tract infection with the potential to cause severe and possibly fatal atypical pneumonia in humans caused by Severe Acute Respiratory Syndrome Coronavirus 2 (SARS-CoV-2), initially named novel coronavirus or 2019-nCoV [1]. The first cases of COVID-19 were reported in Wuhan, China in early December 2019 [2]. Since then, COVID-19 has become a global pandemic due to its transmissibility resulting in it spreading across continents with the number of cases and deaths rising daily [3].

Probe-based confocal laser endomicroscopy (pCLE) is a technique that produces microscopic imaging of living tissue through a fiberoptic miniprobe that can be introduced into the working channel of the bronchoscope [4]. This method could be used in the diagnosis of lung cancer and interstitial lung diseases [5, 6]. We did not find any scientific articles about pCLE images of COVID-19. We introduce a case where
pCLE was used in a patient with suspected lung cancer who had subsequently confirmed COVID-19.

\section{Case Report}

An 87-year-old male who was a non-smoker was admitted to our clinic with complaints of weakness, shortness of breath on exertion, and cough. From May 12, 2020, the patient developed cough and fever up to $38.0^{\circ} \mathrm{C}$ after experiencing hypothermia. Nasopharyngeal swabs for RT-PCR SARS-CoV-2 were negative. CT scans revealed obstruction of the segmental bronchi of the upper lobe of the left lung with development of obstructive pneumonitis and pleurisy (Figure 1). The patient underwent a pleural puncture and $160 \mathrm{~mL}$ of cloudy, bright yellow liquid with reddish loose sediment was evacuated.

The effusion had no signs of malignancy and contained blood elements, lymphocytes, histiocytes, and mesothelial cells.

The patient was hospitalized in the clinic in order to diagnose his pneumonia as being post-ob-

Address for correspondence: Igor Sergeevich Mamenko, Saint-Petersburg State Research Institute of Phthisiopulmonology, Saint-Petersburg, Russian Federation; e-mail:dr.mamenko.is@gmail.com

DOI: 10.5603/ARM.a2021.0067 | Received: 16.11.2020 | Copyright (c) 2021 PTChP | ISSN 2451-4934 | e-ISSN 2543-6031

This article is available in open access under Creative Common Attribution-Non-Commercial-No Derivatives 4.0 International (CC BY-NC-ND 4.0) license, allowing to download articles and share them with others as long as they credit the authors and the publisher, but without permission to change them in any way or use them commercially. 


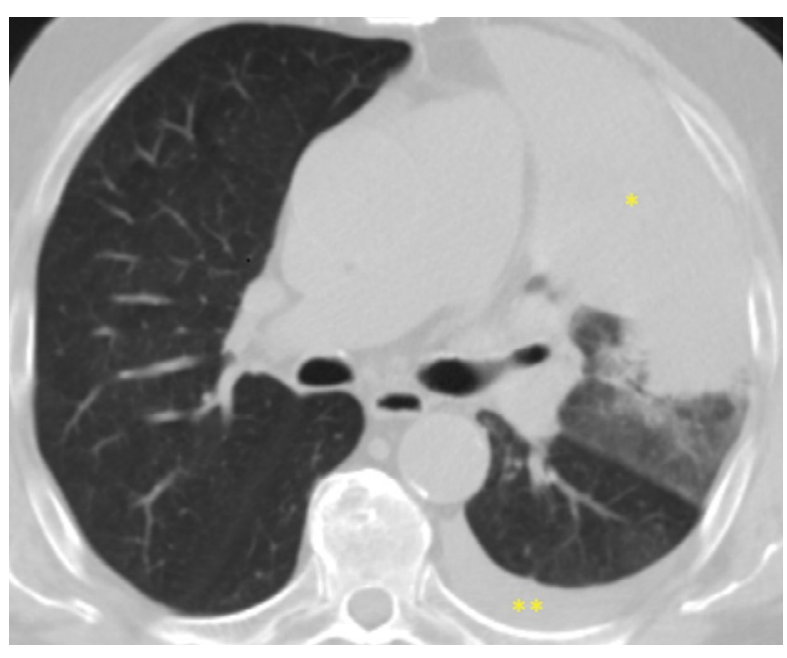

Figure 1. CT-scans showed a large area of consolidation in the left upper lobe $\left({ }^{*}\right)$ and moderate pleural effusion $\left({ }^{* *}\right)$

structive or bacterial/viral in origin. Lung cancer was also suspected.

His comorbidities include coronary artery disease, aortic valve replacement for severe aortic stenosis (2013), superficial thrombophlebitis of the saphenous veins of both legs, type 2 diabetes mellitus with diabetic polyneuropathy and diabetic nephropathy, autoimmune thyroiditis, cholecystectomy (1986), and chronic prostatitis.

After the first day of hospitalization, the patient experienced a sharp deterioration in breathing and a decrease in blood oxygen saturation (89\%) which required artificial ventilation.

Because of the negative smears for RT-PCR SARS-CoV-2 and suspected lung cancer, it was decided to perform bronchoscopy with transbronchial biopsy and confocal laser endomicroscopy.

Bronchoscopy was performed in the intensive care unit by a limited number of staff who donned personal protective equipment (PPE). During bronchoscopy, a moderate amount of liquid-mucous hemorrhagic secretion was noted on both sides, and the bronchus of left segment 3 was obturated with a blood clot. The lumens of the other bronchi were ordinary. pCLE was performed using the Cellvizio system and 1.4mm probe Alveoflex (Mauna Kea Technologies, Paris, France). After removal of the clot, no endobronchial lesions were found. The miniprobe was introduced into all subsegments of the left upper lobe. The pCLE image showed changes in the alveolar frame with increased density up to the point where alveolar structures were absent, thickening of elastic fibers, and a large amount of intra-alveolar secretions (Figure 2). Transbronchial lung biopsy (TBB) was performed from the zones of moderate and severe disorganization of lung tissue by pCLE image data. Also, bronchoalveolar lavage from left upper segmental bronchi for RT-PCR SARS-CoV-2 was performed.

Histological examination revealed signs of diffuse alveolar damage with fibrin, pneumocytes, and macrophages present without any atypical cells (Figure 3). PCR analysis of bronchoalveolar lavage fluid yielded a positive result for SARSCoV-2. Appropriate treatment was started. Unfortunately, the pneumonia evolved and became bilateral. It eventually progressed to respiratory failure and subsequently caused the death of the patient. Postmortem examination did not reveal any signs of malignancy, and the pneumonia was confirmed.

\section{Discussion}

The diagnosis of COVID-19 is based on specific symptoms, history of contact with an infected person, and bilateral lung involvement on CT-scans. The diagnosis is confirmed by a positive nucleic acid test for SARS-CoV-2 commonly from oropharyngeal and nasopharyngeal swabs or bronchoalveolar lavage fluid (BALF) [7]. A nasopharyngeal or oropharyngeal swab for the SARS-CoV-2 RT-PCR test is the primary and preferred method for diagnosis of COVID-19, but its sensitivity varies from $53.6 \%$ to $73.3 \%$ and from $11.1 \%$ to $61.3 \%$, respectively. Their accuracy also depends on how many days have passed since illness onset [8]. Positive rates from BALF in infected patients have been noted in up to $100 \%$ of patients, but all guidelines suggest that bronchoscopy is relatively contraindicated in COVID-19 and/or should play a limited role in diagnosis and management because of the high risk of spreading the infection to the staff involved in the procedure [9]. However, the use of bronchoscopy may be justified if an alternative diagnosis which would change management is suspected [7, 9].

In our case, the diagnosis of COVID-19 was not clear because of the initially unilateral lung infiltrate and pleurisy on CT-scans, and negative nasopharyngeal swab tests. The patient was hospitalized with suspected lung cancer. Probe-based confocal laser endomicroscopy showed density of alveolar thickened fibres, thickening of elastin fibres, and multiple large drops of intraalveolar secretions. In pCLE images, no solid pattern consistent with a malignant disease was noted which allowed us to exclude lung cancer in the differential diagnosis [5]. Histological examina- 


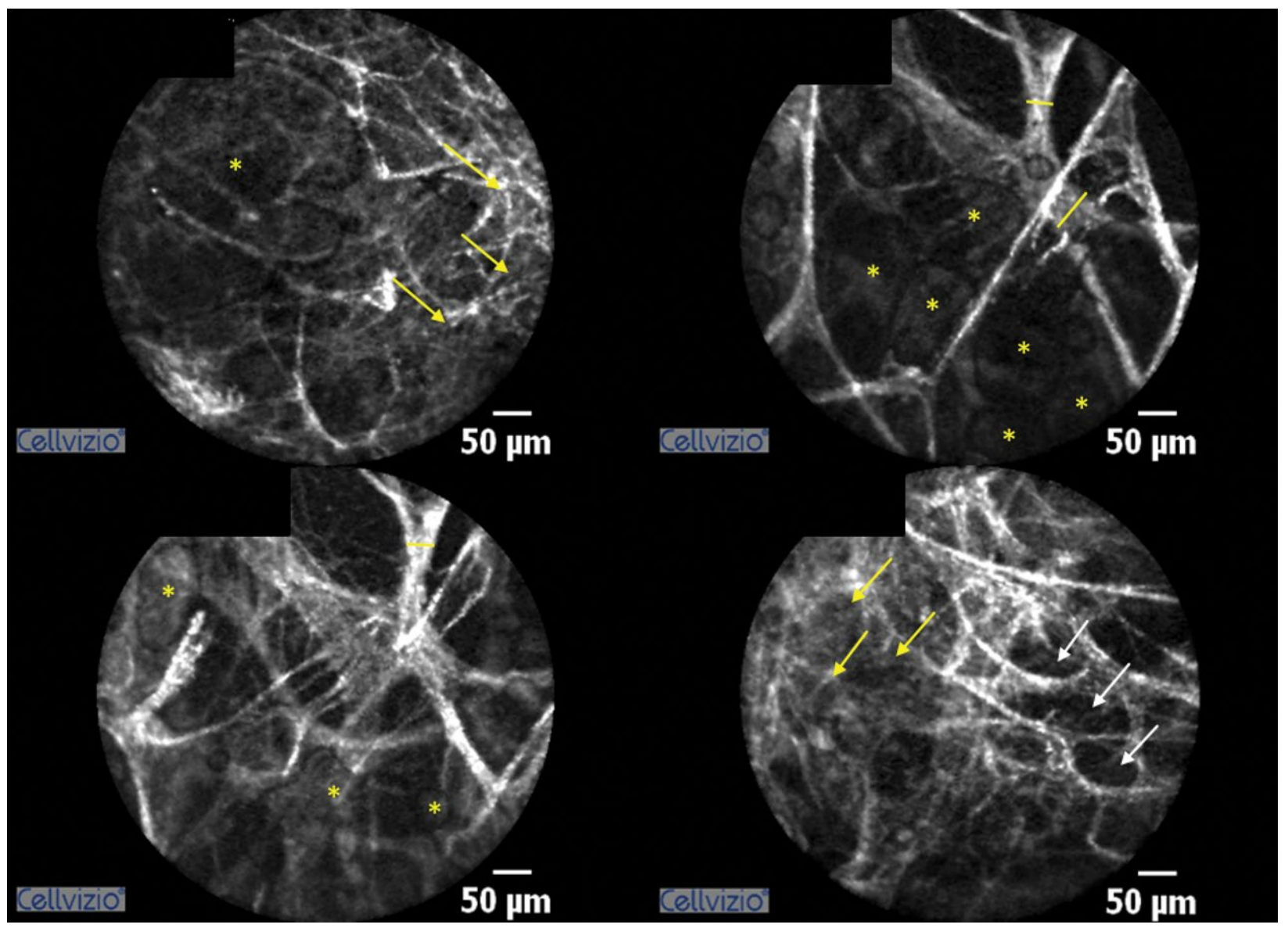

Figure 2. pCLE images of lung tissue of the left upper lobe. Intraalveolar fiber thickness (yellow line), increased density of elastic structures (white arrow) up to disappearance of alveolar structures (yellow arrow), and large drops of intraalveolar secretions (*)

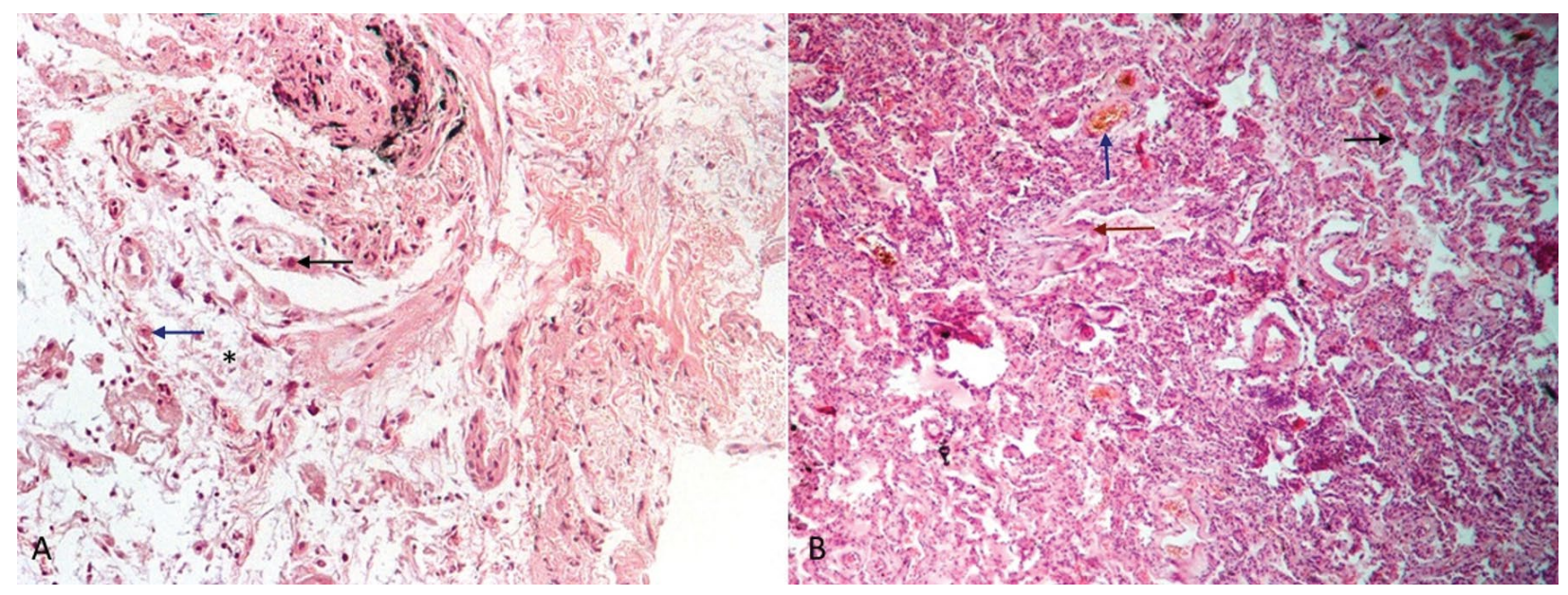

Figure 3. Pathological examination of TBB samples. A. Diffuse alveolar damage, proliferative phase: there is a small amount of pneumocytes in the alveoli (black arrow), macrophages (blue arrow), and fibrin $\left({ }^{*}\right) ; \times 100$, stained with hematoxylin and eosin. B. Diffuse alveolar damage, proliferative phase: accumulation of fibrin in the lumen of the alveoli with polypoid growths of connective tissue (brown arrow), interstitial inflammation with thickening and edema of interalveolar septa (black arrow), and microangiopathy and thrombosis of the vascular bed (blue arrow), stained with hematoxylin and eosin, $\times 40$ 
tion of TBB specimens revealed signs of diffuse alveolar damage described in COVID-19 [10]. To the best of our knowledge, this is the first pCLE image of a patient with COVID-19. This image was not significantly different from other images seen in interstitial lung diseases [6].

\section{Conclusion}

pCLE can be a useful method for suspecting or excluding a diagnosis of COVID-19 in real time if nasopharyngeal swab tests are negative and bronchoscopy is required to establish the diagnosis.

\section{Conflict of interest}

None declared.

\section{References:}

1. Nicola M, O'Neill N, Sohrabi C, et al. Evidence based management guideline for the COVID-19 pandemic - review article. Int J Surg. 2020; 77: 206-216, doi: 10.1016/j.ijsu.2020.04.001, indexed in Pubmed: 32289472.

2. Guan WJ, Ni ZY, Hu Y, et al. China Medical Treatment Expert Group for Covid-19. Clinical characteristics of coronavirus disease 2019 in China. N Engl J Med. 2020; 382(18): 1708-1720, doi: 10.1056/NEJMoa2002032, indexed in Pubmed: 32109013.
3. Bhimraj A, Morgan RL, Shumaker AH, et al. Infectious Diseases Society of America guidelines on the treatment and management of patients with COVID-19. Clin Infect Dis. 2020 [Epub ahead of print], doi: 10.1093/cid/ciaa478, indexed in Pubmed: 32338708.

4. Thiberville L, Moreno-Swirc S, Vercauteren T, et al. In vivo imaging of the bronchial wall microstructure using fibered confocal fluorescence microscopy. Care Med. 2007; 175(1): 22-31, doi: 10.1164/rccm.200605-684OC, indexed in Pubmed: 17023733.

5. Hassan T, Thiberville L, Hermant C, et al. Assessing the feasibility of confocal laser endomicroscopy in solitary pulmonary nodules for different part of the lungs, using either 0.6 or 1.4 mm probes. PLoS One. 2017; 12(12): e0189846, doi: 10.1371/ journal.pone.0189846, indexed in Pubmed: 29267317.

6. Salaün M, Guisier F, Dominique S, et al. In vivo probe-based confocal laser endomicroscopy in chronic interstitial lung diseases: Specific descriptors and correlation with chest CT. Respirology. 2019; 24(8): 783-791, doi: 10.1111/resp.13507, indexed in Pubmed: 30811085.

7. Ora J, Puxeddu E, Cavalli F, et al. Does bronchoscopy help the diagnosis in COVID-19 infection? Eur Respir J. 2020; 56(2): 2001619, doi: 10.1183/13993003.01619-2020, indexed in Pubmed: 32527742.

8. Yang Y, Yang M, Shen C, et al. Evaluating the accuracy of different respiratory specimens in the laboratory diagnosis and monitoring the viral shedding of 2019-nCoV infections. medRxiv. 2020, doi: 10.1101/2020.02.11.20021493.

9. Lentz R, Colt H. Summarizing societal guidelines regarding bronchoscopy during theCOVID-19 pandemic. Respirology. 2020; 25(6): 574-577, doi: 10.1111/resp.13824.

10. Xu Z, Shi L, Wang Y, et al. Pathological findings of COVID-19 associated with acute respiratory distress syndrome. Lancet Respir Med. 2020; 8(4): 420-422, doi: 10.1016/S22132600(20)30076-X, indexed in Pubmed: 32085846. 